\title{
Visualization of Near-Field Enhancements of Gold Triangles by Nonlinear Photopolymerization
}

\author{
Tobias Geldhauser • Shintarou Ikegaya • Andreas Kolloch • Naoki Murazawa • \\ Kosei Ueno • Johannes Boneberg • Paul Leiderer • Elke Scheer • Hiroaki Misawa
}

\begin{abstract}
We report in this paper the near-field distribution in the case of gold triangle arrays by means of two-photon polymerization for a dipole and a quadrupole plasmon mode. In order to link the finite difference in the time domain (FDTD) simulations of the triangle array and the experimental results, extinction spectra for both cases in air and SU-8 environments are shown. In case of the 40-nm thick gold triangles with $85-\mathrm{nm}$ side-length, we show that the calculated and experimentally obtained near-field for the excited dipole mode has the same distribution along the polarization of the exciting laser beam. In case of bigger triangles of 540-nm side-length a quadrupole mode is excited, which leads to a rotation of the near-field distribution by $90^{\circ}$ referred to the polarization of the beam. This effect is also shown in the FDTD simulations.
\end{abstract}

Keywords Near-field · FDTD · Two-photon polymerization · Colloidal lithography · Plasmon resonance

In parallel with the advances in the generation of nanostructures with their interesting electronic [1], chemical [24], and optical properties [5-8], the possibility arose to investigate and use the matter-light interaction on the

T. Geldhauser S. Ikegaya N. Murazawa K. Ueno H. Misawa $(\bowtie)$

Research Institute for Electronic Science, Hokkaido University, Sapporo 001-0021, Japan

e-mail: misawa@es.hokudai.ac.jp

A. Kolloch $\cdot$ J. Boneberg $\cdot$ P. Leiderer $\cdot$ E. Scheer Fachbereich Physik, University of Konstanz, 78457 Konstanz, Germany

K. Ueno

PRESTO, Japan Science and Technology Agency, Kawaguchi 332-0012, Japan nanoscale. A very promising route to overcome the diffraction limit and to focus light on the nanometer scale is plasmonics on metallic nano-objects. A tight focus and huge field enhancements by a factor of $10^{4}$ and more can be achieved and are used to guide light in the area of interest [6]. Prominent examples include surface-enhanced Raman spectroscopy [2-4], scanning near-field optical microscopy [9] and optics on the nanoscale [8]. In all these cases, the optical response of nanoparticles, governed by a collective excitation of conduction electrons and thus the induction of a polarization of the surface charge is used. The generated oscillation of the electron density is referred to as a plasmon and depends on the material, shape, and surrounding medium of the nanoparticle [3, 10-14]. Additionally, electromagnetic coupling between nanoparticles can occur, when they are brought in close vicinity to each other [15-17], which leads to a red-shift of the local surface plasmon resonance $[3,15,16,18]$ and a change in the white-light continuum as Mühlschlegel et al. [19] have shown. In order to further enhance the efficiency of these nanostructures as optical antennae, it is important to investigate the origin of these effects, namely the nearfield distribution around the structures.

The methods to visualize the near-field vary from scanning near-field optical microscopy [20-22], where a tip is used to probe the near-field, to non-interacting methods like photopolymerization [23-28], electron energy loss spectroscopy [29], imprinting of the near-field in phase change materials [30], fluorescence of molecules [31], and direct ablation of the substrate $[5,32]$. In case of the twophoton polymerization a nonlinear two-photon process is used to link polymers together and to form a rigid structure $[33,34]$. Due to this nonlinear process, the polymer is strongly linked where the field intensity is greatest and the loose polymers in the low intensity region are easily 
removed after washing. Thus, only the high-intensity regions remain.

Beside methods like electron-beam lithography $[15,23$, 24, 26-28, 35] and extreme-UV-lithography [36], the colloidal lithography [37] can be used to generate these plasmonic structures $[5,13,18,32]$, offering the advantage of a fast and versatile method to generate large areas of structure arrays with well-defined and sharp edges.

The near-field distribution of nanostructures is mainly simulated by the discrete dipole approximation (DDA) [5, $10,21,29,38,39]$ and finite difference time domain (FDTD) method [24-26, 35, 36, 38] where the propagation of light is computed numerically by solving the Maxwell equations on a pre-defined mesh. Perassi et al. [40] have recently shown that the quality of comparison between experiment and simulations is highly dependent on the transformation of the shape of the nanostructure to the simulations.

Experimental verification of the field enhancement for nanotriangles smaller than $300-\mathrm{nm}$ side-length have been reported by many groups [5, 20, 21, 24, 32]; however, Boneberg et al. [5, 32] have shown by a comparison between direct ablation of triangles in an array and DDA of single excited gold triangles that there is a discrepancy in case of bigger structures. It has been argued that this is due to the influence of the array, which has not been taken into account in the simulations. Recent investigations on single mesoscopic triangles of micrometer side-length by Sajanlal et al. [4] have shown good agreement between the simulations and experiments.

In this paper, we report investigations carried out on gold nanotriangles that have been fabricated by colloidal lithography. We study the behavior of the plasmon resonances and the visualization of the near-field enhancement by means of two-photon polymerization. We chose two different sizes of the gold triangles, $85 \mathrm{~nm}$ and $540 \mathrm{~nm}$ side-length, to excite a dipole and a quadrupole mode. The experimental results are compared with FDTD simulations for an array of nanotriangles in the case of the 85-nm triangles. The properties of the gold triangle with a sidelength of $540 \mathrm{~nm}$ can successfully be described by simulations assuming a dimer configuration of triangles.

\section{Experimental Setup}

The gold triangle arrays used in the experiments have been generated by colloidal lithography [37]. Here, $40 \mathrm{~nm}$ of gold have been evaporated onto a colloidal monolayer to get triangular structures after the removal of the colloids. We have used colloidal particles with $320 \mathrm{~nm}$ and $1.5 \mu \mathrm{m}$ in diameter. These structures have been investigated by means of scanning electron microscopy (SEM), spectros- copy, and two-photon photopolymerization. In order to measure the extinction spectra of the triangle structures, a Hamamatsu photonic multichannel analyzer has been incorporated in a microscope setup to cover the visible and near-infrared region of the spectrum. The infrared measurements have been performed using a Jasco FT/IR6000 TM-M equipped with an infrared microscope attachment. All measurements have been conducted in transmission mode, and the spot size has been $30 \mu \mathrm{m}$ in diameter. The two-photon polymerization was conducted with a 2.9$\mu \mathrm{m}$ thick negative photoresist (SU-8 2002, Microchem. Co) spin-coated on top of the structures and by irradiating the gold triangles with a polarized beam of $800 \mathrm{~nm}$ wavelength and $100 \mathrm{fs}$ pulse width at a repetition rate of $82 \mathrm{MHz}$ from a Spectra Physics Tsunami laser system. The irradiation conditions were $10 \mathrm{~s}$ at an intensity of $480 \mathrm{MW} / \mathrm{cm}^{2}$ for a pulse and a Gaussian profile with a full width half maximum (FWHM) of $18 \mu \mathrm{m}$. Subsequent development and removal of the non-polymerized resist with 2-methoxy1-methylethyl acetate and 2-propanol revealed the polymerized SU-8 due to the optical near-field around the structures. The theoretical investigation of the near-field enhancement and extinction spectra have been conducted by the FDTD method using FDTD Solutions Software provided by Lumerical Inc. and building on previously reported studies $[23,28]$. In the simulations, we used a mesh size of $2 \mathrm{~nm}$ in case of the 85-nm triangles and an array of triangles. The simulations for the $540-\mathrm{nm}$ structures have been conducted for a single triangle and a mesh size of $4 \mathrm{~nm}$. The structures consist of 40-nm thick gold on top of a glass substrate. In order to take into account possible shifts of the local plasmon resonance due to the interaction between the single gold triangles, we have simulated a unit-cell of the gold structures in periodic boundary conditions. The simulated nanostructures have been matched to the triangles used in the experiment (Fig. 1b), especially the rounded tip, and the wedge shape of the border of the structures has been incorporated. For the simulations with SU-8 on top of the structures, a refractive index of 1.56 has been used for the polymer [41], and the dielectric constants of the gold and glass have been taken from Palik [42]. In all simulations, the square of the electric field is shown, normalized to the incident electric field.

\section{Results}

From the characterization of the gold triangles by SEM, as seen in Fig 1a, we infer a distribution of the side-lengths of the gold triangles with $85 \mathrm{~nm}$ with a FWHM of $10 \mathrm{~nm}$. The white-light extinction spectra between $400 \mathrm{~nm}$ and $950 \mathrm{~nm}$ for the gold structures in different environments are shown 

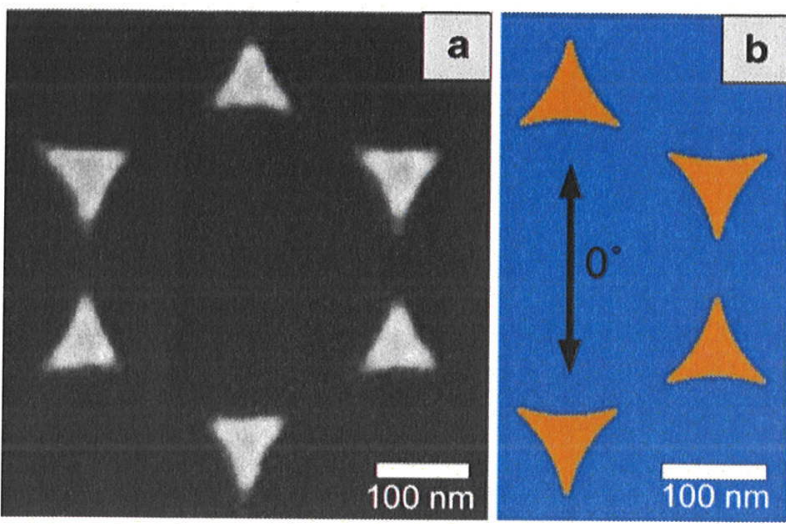

Fig. 1 SEM picture of the gold triangles generated by colloida lithography with $320 \mathrm{~nm}$ polystyrene colloidal particles (a). From this image, the unit-cell of the array for the FDTD simulations has been obtained (b). Here, the $0^{\circ}$ of polarization corresponds to a vertical polarization. The scale bar in both cases is $100 \mathrm{~nm}$

in Fig. 2. In case of an air atmosphere (Fig. 2a), the optical response shows a resonance at $760 \mathrm{~nm}$ with a FWHM of $40 \mathrm{~nm}$ for the two orthogonal polarization directions of the incident light. The polarization direction along one of the symmetry axes of the triangles is denoted as vertical; the orthogonal one, which is parallel to one of the edges, is denoted as horizontal; see Fig. 1b. The calculations by FDTD show a sharper resonance at $740 \mathrm{~nm}$ with a FWHM of $20 \mathrm{~nm}$. When the gold triangles are immersed in the SU-8, the resonance is broadened and shifted to larger wavelength (Fig. 2b). The dipole resonance appears now at $860 \mathrm{~nm}$ (FWHM $80 \mathrm{~nm}$ ) in the experiments and $840 \mathrm{~nm}$ (FHWM $40 \mathrm{~nm}$ ) in the simulations. Superimposed on the plasmon resonance

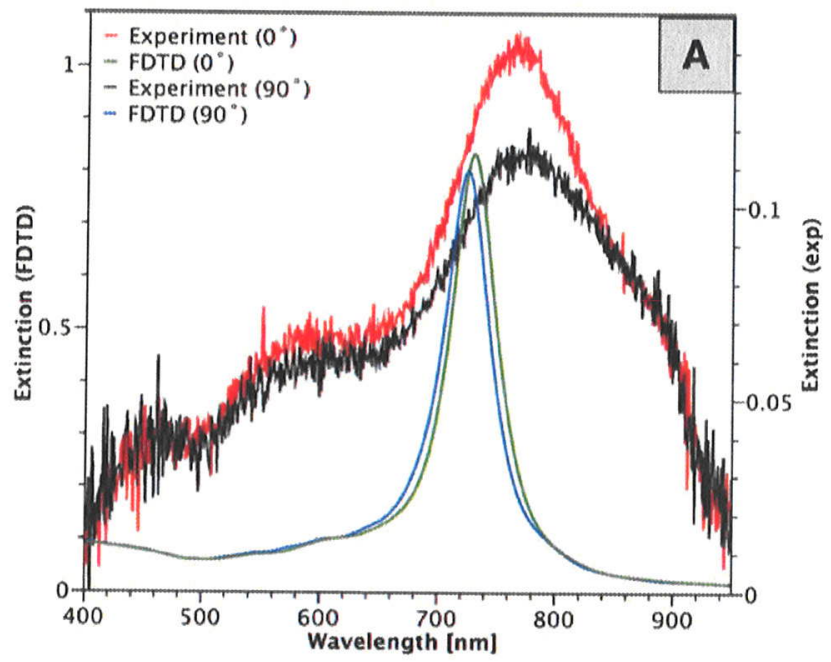

Fig. 2 Experimental and simulated extinction spectra of the $85 \mathrm{~nm}$ gold triangles for vertical and horizontal polarization. The resonance for the triangles in air is at $780 \mathrm{~nm}$ in the experiment and at $740 \mathrm{~nm}$ in the FDTD simulations (a). Covering the structures by SU-8 leads to a signal are interferences due to the polymer layer. They show up as oscillations of the extinction given by the refractive index and the $2.9-\mu \mathrm{m}$ thickness of the polymer layer. These oscillations cannot be seen in the simulations, as we have simulated a bulk SU-8 on top of the nanostructures to keep the simulation times low.

As a next step, we have recorded the image of the nearfield enhancements around the structures for vertical and horizontal polarization using the nonlinear response of the two-photon polymerization induced by irradiation with the $800 \mathrm{~nm}$ laser beam. After irradiation, the samples are rinsed in a solvent which dissolves the non-polymerized parts of the resin. The remaining polymer structures thus correspond to those positions at which the intensity of the light exceeds the threshold to form a rigid network of the SU-8 by two-photon polymerization. The experimental and FDTD results for the near-field of the structures excited by the two orthogonal polarizations of the laser beam is shown in the leftand right-hand side of Fig. 3, respectively. All SEM images were taken at positions well outside of the maximum of the Gaussian laser beam used for the photopolymerization. For the horizontally polarized light, the laser intensity increases from left to right.

In the case of the gold triangles generated by colloids with $1.5 \mu \mathrm{m}$ in diameter, the side-length of the structures is $540 \mathrm{~nm}$, which corresponds to a local surface plasmon resonance for the dipolar mode at $1680 \mathrm{~nm}$ with a FWHM of $150 \mathrm{~nm}$ (Fig. 4c). In the visible light range, a very broad resonance centered at $650 \mathrm{~nm}$ (FWHM $200 \mathrm{~nm}$ ) is observed. The SU-8 on top of the structures shifts the resonance to $680 \mathrm{~nm}$ (FWHM $200 \mathrm{~nm}$ ) and $2000 \mathrm{~nm}$ (FWHM $230 \mathrm{~nm}$ ). The FDTD simulations reveal more

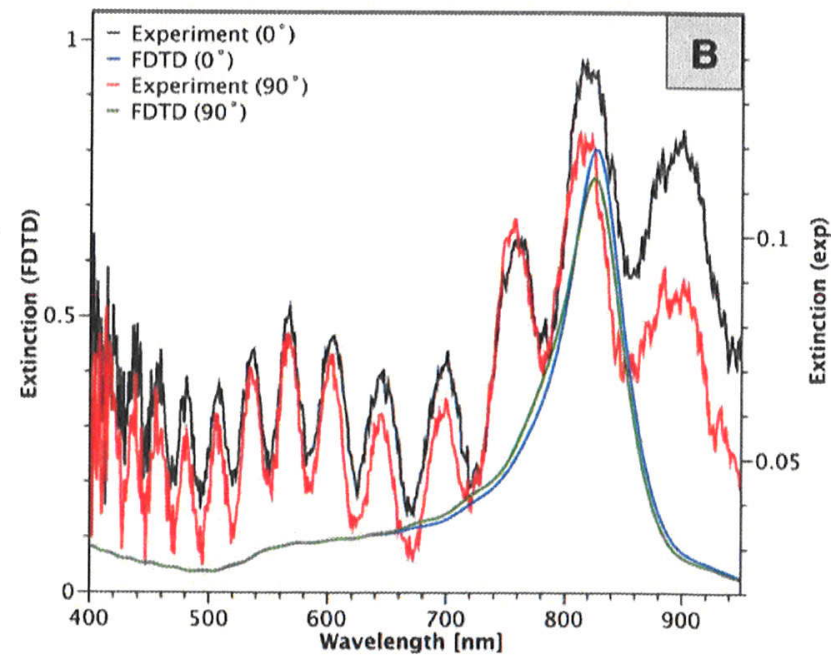

shift of the resonance (b) to $860 \mathrm{~nm}$ and $840 \mathrm{~nm}$, respectively. In both cases, the values for the extinction are shown on the right-hand side for the experiment and on the left for the simulation 

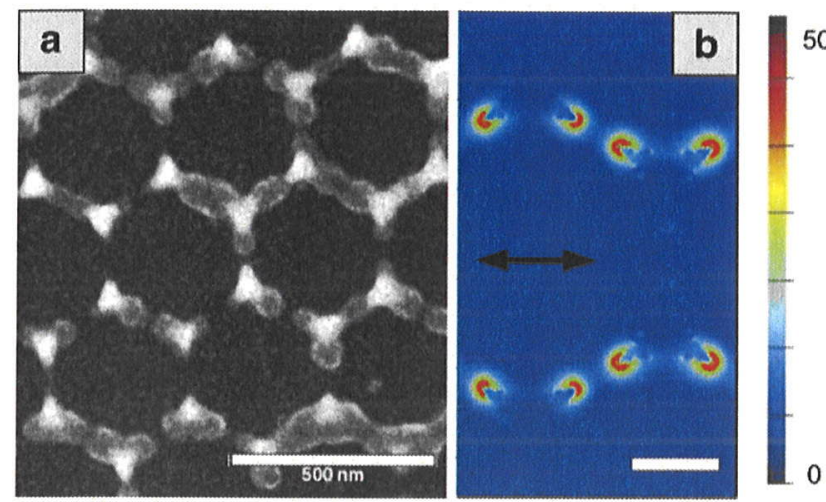

Fig. 3 Visualization of the near-field enhancement on triangles with 85-nm side-length by two-photon polymerization $(\mathbf{a}, \mathbf{b})$ and by FDTD simulations in terms of the square of the electric field $(\mathbf{b}, \mathbf{c})$. The polarization of the $800-\mathrm{nm}$ laser beam was horizontal in panels (a b),
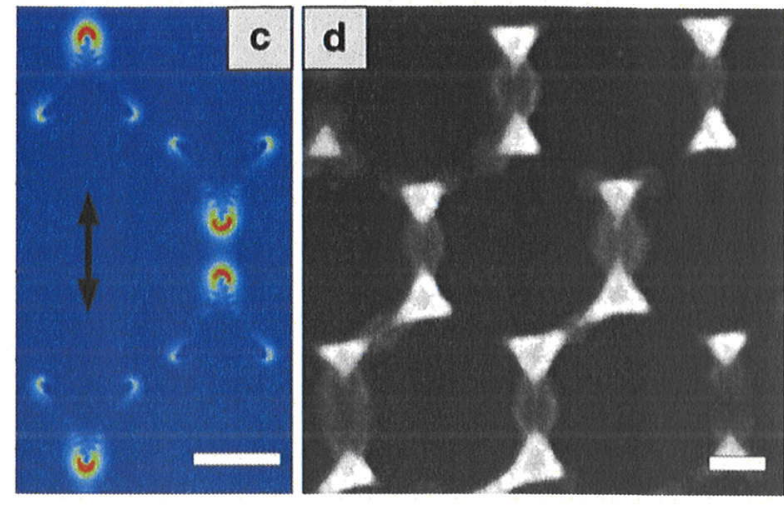

vertical (c, d) as indicated by the arrow. The intensity of the laser beam increases from left to right for the horizontal polarization (a). If not indicated otherwise, the scale bar is $200 \mathrm{~nm}$, and the near-field enhancement is shown in color code for the simulations details in the visible extinction spectra and a very pronounced resonance at $1700 \mathrm{~nm}$ (FWHM $100 \mathrm{~nm}$ ) in air and $2100 \mathrm{~nm}$ (FWHM $150 \mathrm{~nm}$ ) when covered with SU8. The pattern of the polymerized SU-8 after irradiation with a vertically polarized beam is shown in Fig. 4a. Two polymer spots, corresponding to two local maxima of the intensity, appear in the upper edges. Additional maxima in the middle of the base of the lower triangle are observed, but no enhancement at the tips parallel to the polarization direction. In both cases, the near-field enhancement is rotated by $90^{\circ}$ with respect to the polarization of the incident laser light, and no enhancement in between the triangles can be seen. The near-field distribution from the FDTD calculations is shown in Fig. 4b.

\section{Discussion}

The extinction spectra, as shown in Fig. 2, are very sensitive to a variation in shape, gap distance, and other inhomogeneities that are unavoidable with the method of colloidal lithography. One common defect is the bigger structures consisting of several connected triangles [5]. This leads to a broadening and decrease of the maximum of the extinction spectra (Fig. 2), compared with the simulated data and less resolution as the spot size in the measurement covers a large number of unit-cells of the gold triangle structure. Especially in the case of the $540 \mathrm{~nm}$ triangles, the features giving rise to variations of the extinction upon small changes of the wavelength are smeared out. The interferences appearing in the extinction spectra of the
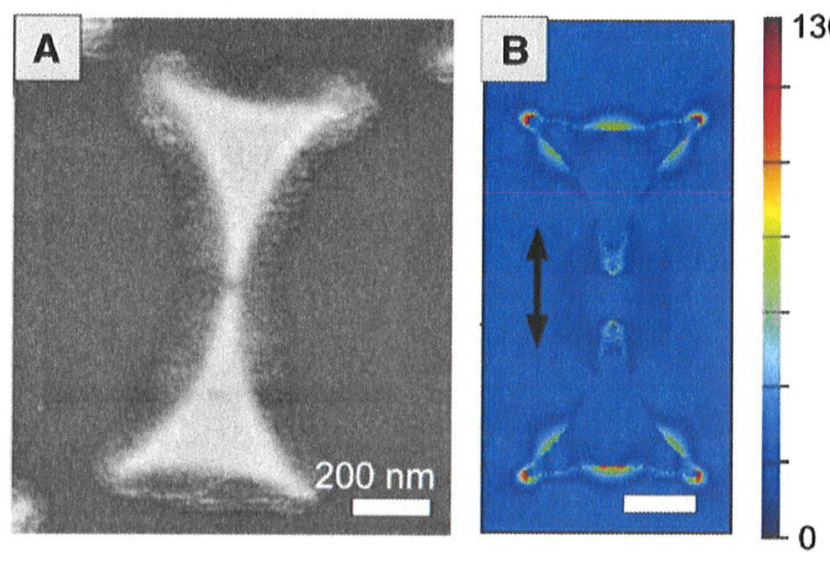

Fig. 4 In the case of gold triangles with 540-nm side-length, the resonance for the local surface plasmon is in the infrared region at $1680 \mathrm{~nm}$ (c). The simulated (b, $780 \mathrm{~nm}$ ) and experimentally (a,
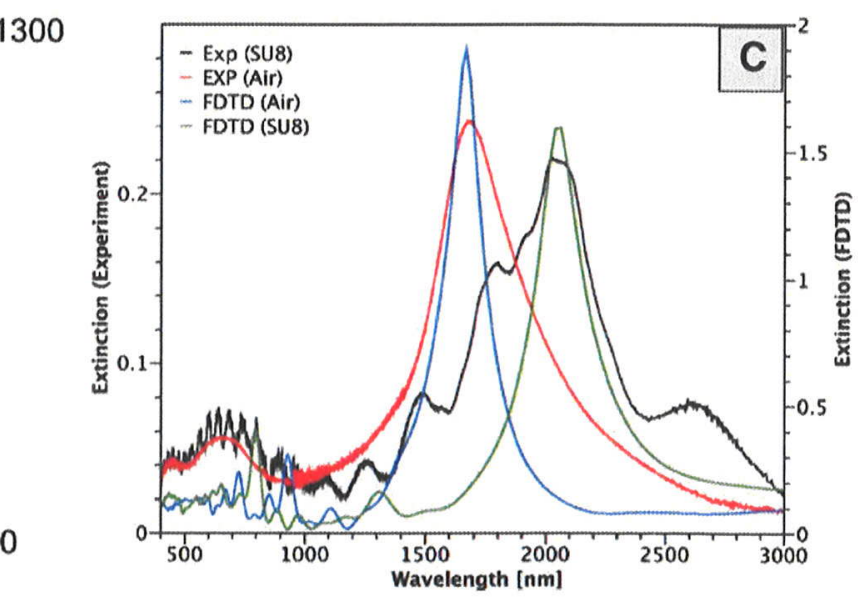

$800 \mathrm{~nm}$ ) obtained near-field enhancement of the gold triangles have been obtained with a vertically polarized laser beam. The scale bar is $200 \mathrm{~nm}$ 
structures covered by the polymer do influence the coupling of light into the nanostructures, but do not alter the nearfield distribution. Despite of the interference, the resonance peak is clearly visible and goes in line with the simulated data for SU-8 described by a refractive index of 1.56. As we can merely excite the dipole resonance at its slope and as a change in size of the triangles will not shift the broad resonance out of the excitation windows for the $800 \mathrm{~nm}$ laser beam, a small variation in size has little effect on the qualitative near-field distribution and will only affect the enhancement factor. This can be seen in Fig. 3 with a very good agreement between the experimental and simulated data for the dipole mode of the local surface plasmon for the gold triangles with 85 -nm side-length.

In the case of the gold triangles with $540-\mathrm{nm}$ sidelength, the agreement between the experimental and theoretical data for the extinction spectra is very good, and in the near-field distribution, the simulations show that the highest intensity of the near-field is rotated by $90^{\circ}$ compared with the polarization of the exciting laser beam. No enhancement is observed in between the triangles. Although the high intensity along the upper side of the triangle is not reproduced by the polymerization experiment in the case of the upper gold triangle, the main features in the upper edges and the adjacent tips can clearly be seen. This at-first-glimpse unexpected pattern can be explained by the excitation of a quadrupole mode of the structure, in contrast to the dipole mode for the smaller triangles with $85-\mathrm{nm}$ side-length. This quadrupole mode causes an extinction peak around $700 \mathrm{~nm}$ (half the wavelength of the dipole mode), as observed in Fig. $4 \mathrm{c}$ in both the experimental and simulated extinction spectra.

\section{Conclusions}

We have studied the near-field enhancement around gold triangles with side-lengths of $85 \mathrm{~nm}$ and $540 \mathrm{~nm}$ by twophoton polymerization. With the help of the extinction spectra, a clear assignment of the plasmon modes was possible. We established a clear correlation between the experimental and theoretical results obtained by FDTD simulations. Especially in the case of bigger triangles, this has proven to be crucial information for the interpretation of the results. For the smaller triangle, very good agreement between the experimental and theoretical results has been obtained. The findings can be described by the excitation of a dipole mode. Consequently, the plasmon mode yields field enhancements parallel to the polarization of the exciting laser beam. For the bigger triangles, a rotation of the field distribution by $90^{\circ}$ between the polarization of the exciting laser beam and the near-field distribution has been obtained in the simulation in excellent agreement with the experimental findings. We demonstrated that this rotation of the near field is caused by the excitation of a quadrupole mode in the bigger structures. These findings strongly suggest that the rotation of the near-field that had been observed before for the case of gold triangles in air in experiment and simulation [5] is caused by the same mechanism. The clarification of this issue is the subject of ongoing investigations.

Acknowledgment This work was supported by the Strategic Japanese-German Cooperative Program of the JST and DFG. H.M. also acknowledges funding from the Ministry of Education, Culture, Sports, Science, and Technology of Japan: KAKENHI Grant-in-Aid for Scientific Research on the Priority Area "Strong Photon-Molecule Coupling Fields" [No. 470 (No. 19049001)] and Grants-in-Aid from Hokkaido Innovation through Nanotechnology Support (HINTS). Support by the DFG Priority Program SPP 1327 (No. Le315/24-1) is also acknowledged.

\section{References}

1. Scheer E, Joyez P, Esteve D, Urbina C, Devoret MH (1997) Conduction channel transmissions of atomic-size aluminum contacts. Phys Rev Lett 78:3535-3538

2. Nie S, Emory SR (1997) Probing single molecules and single nanoparticles by surface-enhanced Raman scattering. Science 275:1102-1106

3. Schuller JA, Barnard S, Wenshan C, Jun YC, White JS Brongersma ML (2010) Plasmonics for extreme light concentration and manipulation. Nat Mater 9:193-204

4. Sajanlal PR, Subramaniam C, Sasanpour P, Rashidian B, Pradeep $T$ (2010) Electric field enhancement and concomitant Raman spectral effects at the edges of a nanometre-thin gold mesotriangle. J Mater Chem 20:2108-2113

5. Boneberg J, König-Birk J, Münzer H-J, Leiderer P, Shuford KL, Schatz GC (2007) Optical near-fields of triangular nanostructures. Appl Phys A 89:299

6. Ropers C, Neacsu CC, Elsaesser T, Albrecht M, Raschke MB, Lienau C (2007) Grating-coupling of surface plasmons onto metallic tips: a nanoconfined light source. Nano Lett 7(9):2784 2788

7. Barnes WL, Dereux A, Ebbesen TW (2003) Surface plasmon subwavelength optics. Nature 424(6950):824-830

8. Suh JY, Donev EU, Lopez R, Feldman LC, Haglund R (2006) Modulated optical transmission of subwavelength hole arrays in metal- $\mathrm{VO}_{2}$ films. Appl Phys Lett 88(13):133115-3

9. Dürig U, Pohl DW, Rohner F (1986) Near-field optical-scanning microscopy. J Appl Phys 59(10):3318-3327

10. Myroshnychenko V, Rodriguez-Fernández J, Pastoriza-Santos I, Funston A, Novo C, Mulvaney P, Liz-Marzán L, Abajo F (2008) Modelling the optical response of gold nanoparticles. Chem Soc Rev 37:1792-1805

11. Wang H, Brandl DW, Le F, Nordlander P, Halas NJ (2006) Nanorice: a hybrid plasmonic nanostructure. Nano Lett 6(4):827 832

12. Sau T, Rogach A, Jäckel F, Klar TA, Feldmann J (2010) Properties and applications of colloidal nonspherical noble metal nanoparticles. Adv Mater 22(16):1805-1825

13. Jensen TR, Malinsky MD, Haynes CL, Van Duyne RP (1999) Nanosphere lithography: tunable localized surface plasmon 
resonance spectra of silver nanoparticles. J Phys Chem B 103 (45):9846 9853

14. Jain PK, El-Sayed MA (2010) Plasmonic coupling in noble metal nanostructures. Chem Phys Lett 487:153-164

15. Rechberger W, Hohenau A, Leitner A, Krenn JR, Lamprecht B, Aussenegg FR (2003) Optical properties of two interacting gold nanoparticles. Opt Commun 220:137-141

16. Jain PK, Huang W, El-Sayed MA (2007) On the Universal scaling behavior of the distance decay of plasmon coupling in metal nanoparticle pairs: a plasmon ruler equation. Nano Lett 7 (7):2080-2088

17. Deutsch B, Hillenbrand R, Novotny L (2010) Visualizing the optical interaction tensor of a gold nanoparticle pair. Nano Lett 10 (2):652-656

18. Merlein J, Kahl M, Zuschlag A, Sell A, Halm A, Boneberg J, Leiderer P, Leitenstorfer A, Bratschitsch R (2008) Nanomechanical control of an optical antenna. Nat Photonics 2:230-233

19. Mühlschlegel P, Eisler H-J, Martin OJF, Hecht B, Pohl DW (2005) Resonant optical antennas. Science 308:1607

20. Hillenbrand R, Keilmann F, Harnap P, Sutherland DS, Aizpurua J (2003) Coherent imaging of nanoscale plasmon patterns with a carbon nanotube optical probe. Appl Phys Lett 83:368

21. Rang M, Jones AC, Zhou F, Li ZY, Wiley BJ, Xia Y, Raschke MB (2008) Optical near-field mapping of plasmonic nanoprisms. Nano Lett 8(10):3357-3363

22. Imura K, Okamoto H, Hossain MK, Kitajima M (2006) Visualization of localized intense optical fields in single goldnanoparticle assemblies and ultrasensitive Raman active sites. Nano Lett 6(10):2173-2176

23. Ueno K, Takabatake S, Nishijima Y, Mizeikis V, Yokota Y, Misawa H (2010) Nanogap-assisted surface plasmon nanolithography. J Phys Chem Lett 1(3):657-662

24. Sundaramurthy A, Schuck PJ, Conley NR, Fromm DP, Kino GS, Moerner WE (2006) Toward nanometer-scale optical photolithography: utilizing the near-field of bowtie optical nanoantennas. Nano Lett 6(3):355-360

25. Ibn El Ahrach H, Bachelot R, Vial A, Lérondel G, Plain J, Royer $P$ (2007) Spectral degeneracy breaking of the plasmon resonance of single metal nanoparticles by nanoscale near-field photopolymerization. Phys Rev Lett 98:107402

26. Ueno K, Juodkazis S, Shibuya T, Mizeikis V, Yokota Y, Misawa H (2009) Nanoparticle-enhanced photopolymerization. J Phys Chem C 113:11720-11724

27. Murazawa N, Ueno K, Mizeikis V, Juodkazis S, Misawa H (2009) Spatially selective nonlinear photopolymerization induced by the near-field of surface plasmons localized on rectangular gold nanorods. J Phys Chem C 113:1147-1149

28. Ueno K, Juodkazis S, Shibuya T, Yokota Y, Mizeikis V, Sasaki K, Misawa H (2008) Nanoparticle plasmon-assisted two-photon polymerization induced by incoherent excitation source. J Am Chem Soc 130:6928-6929

29. Nelayah J, Kociak M, Stephan O, Geuquet N, Henrard L, Garca de Abajo FJ, Pastoriza-Santos I, Liz-Marzn LM, Colliex C (2010) Two-dimensional quasistatic stationary short range surface plasmons in flat nanoprisms. Nano Lett 10(3):902-907

30. Kühler P, García de Abajo FJ, Solis J, Mosbacher M, Leiderer P, Afonso CN, Siegel J (2009) Imprinting the optical near field of microstructures with nanometer resolution. Small 5:1825-1829

31. Koller DM, Hohenester U, Hohenau A, Ditlbacher H, Reil F, Galler N, Aussenegg FR, Leitner A, Trügler A, Krenn JR (2010) Superresolution moire mapping of particle plasmon modes. Phys Rev Lett 104(14):143901

32. Leiderer P, Bartels C, König-Birk J, Mosbacher M, Boneberg J (2004) Imaging optical near-fields of nanostructures. Appl Phys Lett 85:5370

33. Sun Q, Juodkazis S, Murazawa N, Mizeikis V, Misawa H (2010) Freestanding and movable photonic microstructures fabricated by photopolymerization with femtosecond laser pulses. J Micromech Microeng 20:035004

34. Maruo S, Nakamura O, Kawata S (1997) Three-dimensional microfabrication with two-photon-absorbed photopolymerization. Opt Lett 22:132-134

35. Hubert C, Rumyantseva A, Lerondel G, Grand J, Kostcheev S, Billot L, Vial A, Bachelot R, Royer P, Chang SH, Gray SK, Wiederrecht GP, Schatz GC (2005) Near-field photochemical imaging of noble metal nanostructures. Nano Lett 5:615

36. Ekinci Y, Christ A, Agio M, Martin OJF, Solak HH, Löffler JF (2008) Electric and magnetic resonances in arrays of coupled gold nanoparticle in-tandem pairs. Opt Express 16:13287-13295

37. Burmeister F, Schaefle C, Matthes T, Boehmisch M, Boneberg J, Leiderer P (1997) Colloid monolayers as versatile lithographic masks. Langmuir 13:2983-2987

38. Ghosh SK, Pal T (2007) Interparticle coupling effect on the surface plasmon resonance of gold nanoparticles: from theory to applications. Chem Rev 107:4797-4862

39. Shuford KL, Ratner MA, Schatz GC (2005) Multipolar excitation in triangular nanoprisms. J Chem Phys 123:114713

40. Perassi EM, Hernandez-Garrido JC, Moreno MS, Encina ER, Coronado EA, Midgley PA (2010) Using highly accurate 3D nanometrology to model the optical properties of highly irregular nanoparticles: a powerful tool for rational design of plasmonic devices. Nano Lett 10:2097-2104

41. Deubel M, von Freymann G, Wegener M, Pereira S, Busch K, Soukoulis CM (2004) Direct laser writing of three-dimensional photonic-crystal templates for telecommunications. Nat Mater 3:444-447

42. Palik ED, Ghosh G (1991) Handbook of optical constants of solids, 2nd edn. Academic, New York, NY 